\title{
CAR-T cell in vivo tracking method using PET scan with the reporter gene and new investigational tracer $\left[{ }^{18} \mathrm{~F}\right]$ FHBG
}

\author{
Tsubasa Miyazaki ${ }^{1,2}$, Hitoshi Aiyama ${ }^{1,3}$, Eiichi Ishikawa ${ }^{3}$ \\ ${ }^{1}$ Department of Neurosurgery, Graduated School of Comprehensive Human Sciences, University of Tsukuba, Tsukuba, Ibaraki 305-8575, Japan; \\ ${ }^{2}$ Cell-Medicine, Inc., Tsukuba Science City, Ibaraki 305-0047, Japan; ${ }^{3}$ Department of Neurosurgery, Faculty of Medicine, University of Tsukuba, \\ Tsukuba, Ibaraki 305-8575, Japan \\ Correspondence to: Eiichi Ishikawa. Department of Neurosurgery, Faculty of Medicine, University of Tsukuba, 1-1-1 Tennodai, Tsukuba, Ibaraki 305- \\ 8575, Japan. Email: e-ishikawa@md.tsukuba.ac.jp. \\ Provenance: This is an invited Editorial commissioned by the Section Editor Xian-Xin Qiu [Shanghai Proton and Heavy Ion Center (SPHIC), a.k.a. \\ the Proton and Heavy Ion Center of Fudan University Shanghai Cancer Center (FUSCC), Shanghai, China]. \\ Comment on: Keu KV, Witney TH, Yaghoubi S, et al. Reporter gene imaging of targeted T cell immunotherapy in recurrent glioma. Sci Transl Med \\ 2017;9. pii: eaag2196.
}

Submitted May 31, 2017. Accepted for publication Jun 19, 2017.

doi: $10.21037 /$ tcr.2017.06.50

View this article at: http://dx.doi.org/10.21037/tcr.2017.06.50

\section{Development and clinical effects of chimeric antigen receptor (CAR)-T cells}

Recent development of cancer immunotherapy, and CAR-T cell therapy in particular, attracts considerable attention among researchers. In antitumor immunity, cytotoxic $\mathrm{T}$ lymphocytes (CTLs) specifically recognize tumor-associated antigens (TAAs) in an MHC-class I-restricted manner and attack tumor cells. CTL activation and its clonal expansion require co-stimulatory receptors such as $\mathrm{CD} 28,4-1 \mathrm{BB}$ and OX40, and CTL stimulatory cytokine including IL-2 and IFN $\gamma$ in addition to antigen recognition. However, tumor cells escape from the immune surveillance via decreased expression of MHC class 1, increased expression of coinhibitory molecule, inhibitory cytokine production and accumulation of inhibitory immune cells during in vivo growth. CAR gene-designated extracellular binding domain consists of a target-specific antibody or a targeting ligand fused with the intracellular activating CD3zeta chain and the co-stimulatory molecule gene (1). Unlike conventional activated lymphocyte therapy or natural adaptive immunity, genetically engineered CAR-T cells can attack tumor cells in non-MHC class 1-restricted manner without costimulatory molecule signals. Complete remission and durable clinical effect of CD19 CAR-T cell therapy for CD19 positive leukemia are reported in previous studies (2). Treatment strategies using CAR-T cells have also been developed for high-grade gliomas (HGGs). IL-13R $\alpha 2$ is a cell surface receptor, over-expressed in more than a half of HGG patients (3). IL-13 zetakine contains point-mutated IL-13 sequence and it does not bind to IL-13R $\alpha 1$ but specifically binds to IL-13R $\alpha 2$ (4). Therapeutic effects of IL-13 zetakine-transfected $\mathrm{T}$ cells have been reported in in vitro and in vivo studies using several cell lines containing a tumor stem cell line and isolated HGG cells $(5,6)$. A case study of HGG patients treated with IL-13 zetakine transfected $\mathrm{T}$ cells showed safety of the cell injection and efficacy during clinical monitoring (7). In this study, CTLs, genetically modified to express the herpes simplex virus 1 thymidine kinase (HSV1-tk) suicide gene, were injected in HGG patients, and HSV1-tk positive CTLs were detected by the PET scan with the uptake of a ${ }^{18} \mathrm{~F}$ radiolabelled 9-[4$\left[{ }^{18} \mathrm{~F}\right]$ fluoro-3-(hydroxymethyl)butyl $]$ guanine $\left(\left[{ }^{18} \mathrm{~F}\right] \mathrm{FHBG}\right)$ as a probe.

\section{Determination of treatment effect-problems of prognosis estimation}

Easy and exact method to predict therapeutic effect of these immunotherapies and patient prognosis has not been found to date. In general, detection of the delayed-type hypersensitivity (DTH) reaction using TAA is performed after immunotherapy. Also in our study, DTH-positive 
patients have been found to experience a good prognosis (8). A recent study shows the correlation between preoperative PD-L1 expression of circulating mononuclear cells and prognosis after immunotherapy, and that is expected as a prognostic marker for immunotherapy (9). However, they are not real-time tools for evaluating treatment effect during immunotherapy. MR imaging is a repeatable imaging tool for detection of the treatment effect. Response evaluation criteria in solid tumors (RECIST) based on MR imaging are most frequently used for evaluation of the local reaction of antitumor therapy including chemotherapy. However, the use of these criteria for immunotherapy has some issues such as underestimation of progression-free survival due to pseudoprogression caused by the cell influx into the tumor microenvironment and no correlation with long-term prognosis (10). Although immune related response criteria (irRC) were advocated to solve these issues (11), it takes 2-3 months for the first response assessment to determine the therapeutic effect, and patients lose treatment opportunity if true progression occurs during this period. For these reasons, development of a method of immune cells tracking and early therapeutic effect prediction has been urged. This non-invasive in vivo imaging of dynamics of immune cells in the human body has become available in clinical practice with the development of several new molecular imaging methods. It will be an important method to improve development and optimization of immunotherapy.

\section{CTL Tracking using [ $\left.{ }^{1} 8 F\right]$ FHBG}

$\left[{ }^{18} \mathrm{~F}\right]$ FHBG is a PET tracer with an ${ }^{18} \mathrm{~F}$-labelled side chain of penciclovir used for detecting HSV1-tk or HSVsr39tk mutant protein $(12) \cdot\left[{ }^{18} \mathrm{~F}\right]$ FHBG is not degraded in mammalian TK and does not emit a radio signal. In preclinical models using rats and rabbits, $\left[{ }^{18} \mathrm{~F}\right] \mathrm{FHBG}$ showed to be safe after administration in doses 100 times higher than for humans, and it was approved by FDA as an investigational new drug (13). Yaghoubi et al. also studied human pharmacokinetics and dosimetry of $\left[{ }^{18} \mathrm{~F}\right]$ FHBG with ten healthy volunteers and showed no toxicity (14). The safety and feasibility of radiolabeled $\left[{ }^{18} \mathrm{~F}\right]$ FHBG PET is studied in several clinical fields including recurrent glioma $(7,15)$.

Keu et al. reported the in vivo kinetics of CAR-T cells detected by PET scan using $\left[{ }^{18} \mathrm{~F}\right]$ FHBG (16). Genetically engineered IL-13 zetakine CAR-T cells were transfected with hygromycin phosphotransferases/HSV-tk fused in- frame gene and glucocorticoid resistance locus. Those CAR-T cells were positively selected using hygromycin $\mathrm{B}$ and indirect labeling with $\left[{ }^{18} \mathrm{~F}\right]$ FHBG. In addition, glucocorticoid receptor resistance locus allowed using that CAR-T cells in HGG patients during steroid therapy. Those sequences were transfected into CTL by electroporation using plasmid DNA. In this Keu's study, 7 patients including the first patient who administrated $\left[{ }^{18} \mathrm{~F}\right]$ FHBG with relapsed/recurrent HGG were enrolled. These patients received autologous or allogeneic CAR-T cells, and in vivo monitoring was performed by the $\left[{ }^{18} \mathrm{~F}\right]$ FHBG-PET scan. Safety and stability of CAR-T cells and $\left[{ }^{18} \mathrm{~F}\right]$ FHBG were examined. Rickham reservoir/catheter was installed to reach the tumor resection site after surgery or biopsy and CAR-T cells were injected directly into the tumor site. Patients No. 1-2 administered autologous CAR-T cells and the patients No. 3-7 administered allogeneic CAR-T cells (GRm13Z40-2 CTL line) derived from healthy donors. Patients No. 3-7 simultaneously administered recombinant human IL-2. More than 99\% of the produced CAR-T cells were CD 8 positive $\mathrm{T}$ cells, and $\left[{ }^{18} \mathrm{~F}\right]$ FHBG uptake of CAR-T cells was significantly higher than that of the parental cells. After the $\left[{ }^{18} \mathrm{~F}\right]$ FHBG uptake, CAR-T cells retained proliferation ability for 2 days.

One patient had rash and redness in the lower part of the face after administration of FHBG, but the symptoms improved after administration of diphenhydramine. There were no serious adverse events other than in this patient. After administration of $\left[{ }^{18} \mathrm{~F}\right]$ FHBG, the $\left[{ }^{18} \mathrm{~F}\right]$ FHBG accumulated in the tumor area and tumor resection site were observed. One patient whose tumor was attached to the dura matter with rich tumor blood vessels showed 3-4 times higher accumulation of $\left[{ }^{18} \mathrm{~F}\right]$ FHBG compared to the baseline. Since $\left[{ }^{18} \mathrm{~F}\right]$ FHBG cannot pass through the BBB, $\left[{ }^{18} \mathrm{~F}\right]$ FHBG accumulated in areas rich in tumor blood vessels without the BBB. PET scan data calculation methods need to be validated, however, an increase in signal was observed after the CAR-T cells administration in all patients except Patient 1 , who could not receive preCAR-T cell injection PET scan. Furthermore, in patients with multiple tumors an increase in $\left[{ }^{18} \mathrm{~F}\right]$ FHBG signal was observed in areas where CTLs were not injected, suggesting transition of CAR-T cells between tumors. These data proved that PET scan with $\left[{ }^{18} \mathrm{~F}\right] \mathrm{FHBG}$ can visualize HSV1tk expressing CAR-T cells accumulated in the tumor site after injection. Data on safety of $\left[{ }^{18} \mathrm{~F}\right]$ FHBG needs to be collected in the future. In this study, the compound targeted recurrent gliomas, and most patients died within 12 months 
after administration of CAR-T cells. Further research will be focused on methods to evaluate the therapeutic effect of CAR-T cell therapy.

\section{Acknowledgements}

None.

\section{Footnote}

Conflicts of Interest: The authors have no conflicts of interest to declare.

\section{References}

1. Sengupta S, Mao G, Gokaslan ZS, et al. Chimeric antigen receptors for treatment of glioblastoma: a practical review of challenges and ways to overcome them. Cancer Gene Ther 2017;24:121-9.

2. Maude SL, Frey N, Shaw PA, et al. Chimeric antigen receptor $\mathrm{T}$ cells for sustained remissions in leukemia. $\mathrm{N}$ Engl J Med 2014;371:1507-17.

3. Brown CE, Warden CD, Starr R, et al. Glioma IL13R $\alpha 2$ is associated with mesenchymal signature gene expression and poor patient prognosis. PLoS One 2013;8:e77769.

4. Debinski W, Gibo DM, Obiri NI, et al. Novel anti-brain tumor cytotoxins specific for cancer cells. Nat Biotechnol 1998;16:449-53.

5. Kahlon KS, Brown C, Cooper LJ, et al. Specific recognition and killing of glioblastoma multiforme by interleukin 13-zetakine redirected cytolytic $\mathrm{T}$ cells. Cancer Res 2004;64:9160-6.

6. Brown CE, Starr R, Aguilar B, et al. Stem-like tumorinitiating cells isolated from IL13R $\alpha 2$ expressing gliomas are targeted and killed by IL13-zetakine-redirected T Cells. Clin Cancer Res 2012;18:2199-209.

7. Yaghoubi SS, Jensen MC, Satyamurthy N, et al. Noninvasive detection of therapeutic cytolytic $\mathrm{T}$ cells with 18F-FHBG PET in a patient with glioma. Nat Clin Pract Oncol 2009;6:53-8.

Cite this article as: Miyazaki T, Aiyama H, Ishikawa E. CAR-T cell in vivo tracking method using PET scan with the reporter gene and new investigational tracer $\left[{ }^{18} \mathrm{~F}\right] \mathrm{FHBG}$. Transl Cancer Res 2017;6(Suppl 6):S1003-S1005. doi: 10.21037/ tcr.2017.06.50
8. Ishikawa E, Muragaki Y, Yamamoto T, et al. Phase I/ IIa trial of fractionated radiotherapy, temozolomide, and autologous formalin-fixed tumor vaccine for newly diagnosed glioblastoma. J Neurosurg 2014;121:543-53.

9. Bloch O, Lim M, Sughrue ME, et al. Autologous Heat Shock Protein Peptide Vaccination for Newly Diagnosed Glioblastoma: Impact of Peripheral PD-L1 Expression on Response to Therapy. Clin Cancer Res 2017. [Epub ahead of print].

10. Huang RY, Neagu MR, Reardon DA, et al. Pitfalls in the neuroimaging of glioblastoma in the era of antiangiogenic and immuno/targeted therapy - detecting illusive disease, defining response. Front Neurol 2015;6:33.

11. Wolchok JD, Hoos A, O'Day S, et al. Guidelines for the evaluation of immune therapy activity in solid tumors: immune-related response criteria. Clin Cancer Res 2009;15:7412-20.

12. Alauddin MM, Conti PS. Synthesis and preliminary evaluation of 9-(4-[18F]-fluoro-3-hydroxymethylbutyl) guanine ([18F]FHBG): a new potential imaging agent for viral infection and gene therapy using PET. Nucl Med Biol 1998;25:175-80.

13. Yaghoubi SS, Couto MA, Chen CC, et al. Preclinical safety evaluation of $18 \mathrm{~F}-\mathrm{FHBG}$ : a PET reporter probe for imaging herpes simplex virus type 1 thymidine kinase (HSV1-tk) or mutant HSV1-sr39tk's expression. J Nucl Med 2006;47:706-15.

14. Yaghoubi S, Barrio JR, Dahlbom M, et al. Human pharmacokinetic and dosimetry studies of [(18)F]FHBG: a reporter probe for imaging herpes simplex virus type1 thymidine kinase reporter gene expression. J Nucl Med 2001;42:1225-34.

15. Eissenberg LG, Rettig MP, Ritchey JK, et al. [18F]FHBG PET/CT Imaging of CD34-TK75 Transduced Donor T Cells in Relapsed Allogeneic Stem Cell Transplant Patients: Safety and Feasibility. Mol Ther 2015;23:1110-22.

16. Keu KV, Witney TH, Yaghoubi SS, et al. Reporter gene imaging of targeted $\mathrm{T}$ cell immunotherapy in recurrent glioma. Sci Transl Med 2017;9. pii: eaag2196. 